\title{
Surveillance and Care of the Gynecologic Cancer Survivor
}

\author{
Stephanie S. Faubion, MD, Kathy L. MacLaughlin, MD. ${ }^{2}$ Margaret E. Long, MD, ${ }^{3}$ \\ Sandhya Pruthi, MD, ${ }^{4}$ and Petra M. Casey, $\mathrm{MD}^{3}$
}

Abstract

Background: Care of the gynecologic cancer survivor extends beyond cancer treatment to encompass promotion of sexual, cardiovascular, bone, and brain health; management of fertility, contraception, and vasomotor symptoms; and genetic counseling.

Methods: This is a narrative review of the data and guidelines regarding care and surveillance of the gynecologic cancer survivor. We searched databases including PubMed, Cochrane, and Scopus using the search terms gynecologic cancer, cancer surveillance, and cancer survivor and reached a consensus for articles chosen for inclusion in the review based on availability in the English language and publication since 2001, as well as key older articles, consensus statements, and practice guidelines from professional societies. However, we did not undertake an extensive systematic search of the literature to identify all potentially relevant studies, nor did we utilize statistical methods to summarize data. We offer clinical recommendations for the management of gynecologic cancer survivors based on review of evidence and our collective clinical experience.

Results: Key messages include the limitations of laboratory studies, including CA-125, and imaging in the setting of gynecologic cancer surveillance, hormonal and non-hormonal management of treatment-related vasomotor symptoms and genitourinary syndrome of menopause, as well as recommendations for general health screening, fertility preservation, and contraception.

Conclusions: A holistic approach to care extending beyond cancer treatment alone benefits gynecologic cancer survivors. In addition to surveillance for cancer recurrence and late treatment side effects, survivors benefit from guidance on hormonal, contraceptive, and fertility management and promotion of cardiovascular, bone, brain, and sexual health.

\section{Introduction}

G YNECOLOGIC MALIGNANCIES MAY INVOLVE the uterine cervix, uterine corpus, ovary, vulva, and vagina. As a group, gynecologic cancers are the third most common cancers and causes of cancer deaths in women in the United States (U.S.) after breast and lung malignancies. ${ }^{1}$ Five and a half percent of all new cancer diagnoses in the U.S. are gynecologic, with over a million U.S. women living with a gynecologic cancer diagnosis each year. ${ }^{2-5}$ Due to the high 5year survival rate $->65 \%$ for most gynecologic cancersmany women need longer-term follow-up in primary care settings. ${ }^{2-5}$ In addition to surveillance for recurrence, women benefit from provider attention to hormonal management, vasomotor symptom control, osteoporosis prevention, car- diovascular and brain health, fertility, contraception, sexual function, and genetic testing. This review focuses on clinical management of gynecologic cancer survivors in terms of available evidence and recommendations.

\section{Epidemiology of Gynecologic Cancers}

Cancers in gynecologic organs vary in their epidemiologies. $^{2-5}$ Table 1 summarizes data regarding gynecologic cancers in the United States.

Most endometrial cancers thrive in an estrogen-dominated hormonal milieu, as seen with obesity, polycystic ovarian syndrome, and nulliparity. They are generally diagnosed early given associated vaginal bleeding. ${ }^{2}$ Oral contraceptives and other progestin therapies lower risk in a time-dependent

\footnotetext{
${ }^{1}$ Division of General Internal Medicine, ${ }^{2}$ Department of Family Medicine, ${ }^{3}$ Department of Obstetrics and Gynecology, and ${ }^{4}$ Division of General Internal Medicine, Breast Diagnostic Clinic, Women's Health Clinic, Mayo Clinic, Rochester, Minnesota.

(C) Stephanie S. Faubion et al. 2015; Published by Mary Ann Liebert, Inc. This Open Access article is distributed under the terms of the Creative Commons Attribution Noncommercial License (http://creativecommons.org/licenses/by-nc/4.0/) which permits any noncommercial use, distribution, and reproduction in any medium, provided the original author(s) and the source are credited.
} 
Table 1. Summary of Data for Gynecologic Cancers in the United States

\begin{tabular}{|c|c|c|c|c|}
\hline \multirow[b]{2}{*}{ Categories } & \multicolumn{4}{|c|}{ Site of cancer } \\
\hline & Cervix ${ }^{2}$ & Endometrium $^{3}$ & Ovary $^{4}$ & Vulva $a^{5}$ \\
\hline New cases per 100,000 women & 7.8 & 24.6 & 12.3 & 2.4 \\
\hline Deaths per 100,000 women & 2.3 & 4.3 & 7.9 & 0.5 \\
\hline Five-year survival & $67.9 \%$ & $81.5 \%$ & $44.6 \%$ & $70.5 \%$ \\
\hline Women living with disease (estimated) & 249,632 & 610,804 & 188,867 & - \\
\hline Lifetime risk of occurrence & $0.7 \%$ & $2.7 \%$ & $1.3 \%$ & $0.3 \%$ \\
\hline Median age in years at diagnosis & 49 & 62 & 63 & 68 \\
\hline Median age in years at death & 57 & 71 & 71 & 78 \\
\hline Trend in diagnoses per year & $-1.2 \%$ & Stable & $-1.0 \%$ & $+0.5 \%$ \\
\hline Trend in deaths per year & $-1.3 \%$ & $+1.0 \%$ & $-2.0 \%$ & $+0.7 \%$ \\
\hline
\end{tabular}

manner. The effect may persist for 15 years after progestin discontinuation. ${ }^{6}$ While non-Hispanic white women are more likely to be diagnosed, black women are more likely to die of endometrial cancer. Women with hereditary nonpolyposis colon cancer are diagnosed at a younger age (average age 47 years), and have a $20 \%-60 \%$ risk of occurrence by age 70 years. $^{2}$

Though rare, with a lifetime risk of occurrence of $1.3 \%,{ }^{4}$ ovarian cancer is the most deadly of the gynecologic cancers, as it tends to be metastatic at diagnosis. Risk of ovarian cancer is reduced by pregnancy, lactation, and oral contraceptive use, wherein ovulation does not occur. Women with BRCA 1 or 2 mutations have lifetime ovarian cancer risks of $39 \%$ and $11 \%-17 \%$ respectively. ${ }^{7}$ Recent data suggest that serous epithelial ovarian cancers start in the fallopian tube. ${ }^{8}$ The highest disease and death rates occur in non-Hispanic white women. ${ }^{4}$ The vast majority of cervical, vulvar, and vaginal cancers are related to human papilloma virus (HPV), especially types 16 and 18. Immunocompromised status, black or Hispanic ethnicity, and smoking are associated with higher rates of these cancers. Cervical cytology and high-risk HPV screening has led to a dramatic decline in cervical cancer incidence. Over half of cervical cancers diagnosed in the U.S. are due to lack of screening. ${ }^{9}$

A common definition of survivorship is the process of living with, through, and beyond cancer. By this definition, cancer survivorship begins at diagnosis and includes individuals who continue treatment, those who are focused on recurrence risk reduction, and those managing chronic disease. ${ }^{10}$

Surveillance provides an opportunity to educate women about concerning signs and symptoms, to address relevant quality of life issues, as well as to provide preventive health services and counseling. ${ }^{11}$ This approach aligns with the National Cancer Institute definitions of survivorship and needs of cancer survivors.

Treatment of the whole woman includes not only a review of follow-up care and a wellness plan, but also discussions regarding adjustment to physical and emotional changes after cancer treatment, coping with the fear of recurrence, and family issues or concerns.

\section{Gynecologic cancer surveillance}

Surveillance after gynecologic malignancy treatment aims to detect recurrence when a meaningful and cost-effective intervention for quality of life or life expectancy improvement is possible. The lack of high-quality, evidence-based guide- lines impacts the surveillance of gynecologic malignancy survivors. In 2011, the Society of Gynecologic Oncologists (SGO) published the most comprehensive recommendations to date but acknowledged that they are based primarily on retrospective studies and expert opinion and emphasized the need for future prospective studies. ${ }^{11}$ In their guideline publication, the SGO included an outline for clinicians to use at surveillance visits to ensure appropriate follow up (Fig. 1).

For early surveillance, the SGO recommends follow-up in an oncology practice. Later, follow-up can be with a primary care provider once a woman is on an annual visit schedule. There are no clear recommendations for routine testing during later follow-up unless there is suspicion of recurrence. Annual cervical or vaginal cytology may be useful in subsequent follow-up of cervical, vulvar, and vaginal cancers. The timing of transition from early to late follow-up varies with tumor type and stage and is detailed below, along with recommendations for early follow-up.

\section{Endometrial cancer surveillance}

The SGO checklist bases systems review and physical examination frequency on cancer stage at diagnosis and the interval since diagnosis. Stage 1A grade 1 or 2 endometrial cancer places the patient in a lower risk category, with a visit suggested every 6 months for the first year and annually thereafter. Intermediate risk patients with stage $1 \mathrm{~B}-2$ cancer are seen every 3 months for the first year, every 6 months until 5 years after diagnosis, and then annually. Stage 3-4 or serous or clear cell endometrial cancer survivors fall in the highest risk category and are seen every 3 months for 2 years, every 6 months for 3 years, and annually thereafter. The SGO does not recommend vaginal cytology or routine testing for cancer antigen 125 (CA-125) levels. While the SGO does not recommend imaging for surveillance of asymptomatic women, the group suggests computed tomography (CT), positron emission tomography (PET), or both if recurrence is suspected based on symptoms or examination. ${ }^{11}$ The American College of Radiology agrees with the lack of evidence to support imaging asymptomatic women but suggests both CT and PET be performed if recurrence is clinically suspected. ${ }^{12}$ Testing for CA-125 may be considered in women with symptoms, examination findings, higher risk stages or histologic types of endometrial cancer, or in women with an elevated CA-125 pretreatment. ${ }^{11}$

A 2010 review of post-endometrial cancer surveillance studies reported similar patient outcomes despite different 


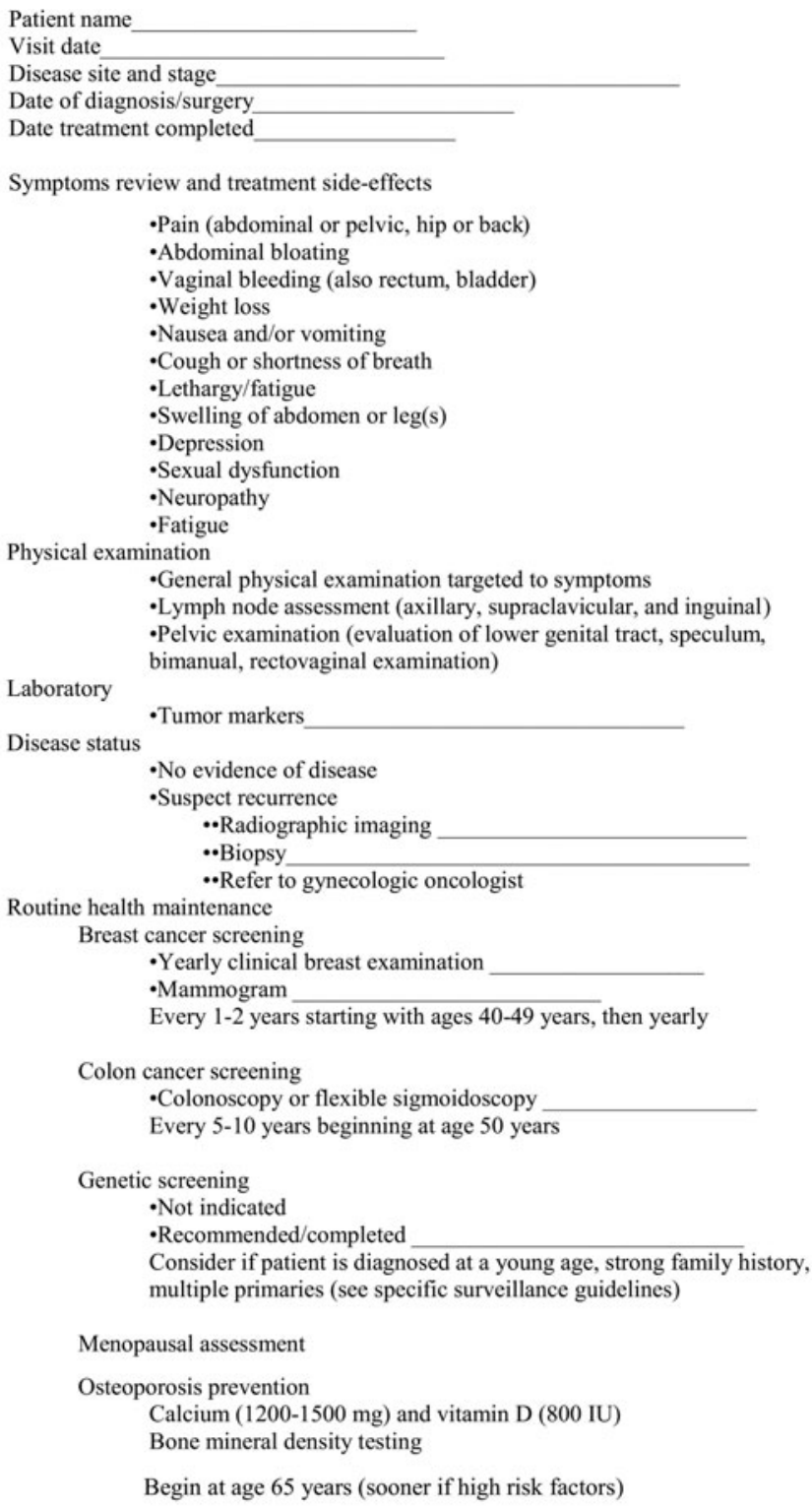

FIG. 1. Society of Gynecologic Oncologists surveillance checklist. Reprinted from the American Journal of Obstetrics and Gynecology, Vol. 204, Issue 6; Salani R, Backes FJ, Fung MF, Holschneider CH, Parker LP, Bristow RE, Goff BA; Posttreatment surveillance and diagnosis of recurrence in women with gynecologic malignancies: Society of Gynecologic Oncologists recommendations; pages 466-478, 2011; with permission from Elsevier.

surveillance protocols. Survival rates were similar in women with asymptomatic and symptomatic recurrence. Physical examination, including bimanual and recto-vaginal examination, was reported as more effective than vaginal cytology, laboratory testing, or imaging to detect recurrence. ${ }^{13}$

\section{Ovarian cancer surveillance}

For patients with a history of epithelial ovarian cancer, the SGO recommends systems review and physical examination every 3 months for the first 2 years, every 4 to 6 months for the third year, every 6 months for the fourth and fifth years, and annually after 5 years. As with endometrial cancer survivors, there is no indication for vaginal cytology if hysterectomy has been performed, or for imaging in asymptomatic patients. ${ }^{11}$ Measurement of CA-125 is optional, as no survival advantage has been demonstrated in women whose recurrence was diagnosed by CA- 125 as compared with those diagnosed clinically. ${ }^{14}$ If clinical recurrence is suspected, CT, PET, or both and CA-125 testing are recommended. ${ }^{11}$

Human epididymis protein 4 (HE4) is a newer, U.S. Food and Drug Administration (FDA)-approved serum biomarker for monitoring disease progression or recurrence in women with epithelial ovarian cancer. In a 2014 review of seven biomarker studies, the authors concluded that although HE4 appears to be an earlier recurrence predictor than CA-125 and may have prognostic advantages, additional study is needed. Further, they questioned whether earlier recurrence detection impacts survival or quality of life. ${ }^{15}$

Surveillance frequency for systems review and physical examination is increased for nonepithelial ovarian cancer (germ cell or sex cord-stromal tumors) to every 2-4 months for the first 2 years, then every 6 months for sex cord-stromal tumors, but yearly for germ cell tumors. The presence of tumor marker elevation at the time of diagnosis determines which markers (e.g., alpha fetoprotein, human chorionic gonadotropin, inhibin, lactate dehydrogenase, or others) will be helpful for detecting recurrence. The appropriate serum tumor markers are indicated every 2-4 months for the first 2 years after diagnosis of both nonepithelial ovarian cancer types, but continue after that at 6-month intervals only for those with sex-cord stromal tumors. During the first 2 years when most recurrences occur, CT rather than tumor markers may be useful in women with germ cell tumors and normal tumor markers at diagnosis. However, imaging is not indicated for asymptomatic women with sex cord-stromal tumors. For clinically-suspected recurrence of nonepithelial ovarian cancer, CT and serum tumor markers are indicated. ${ }^{11}$

\section{Cervical cancer surveillance}

The SGO recommends that women with a history of cervical cancer have systems review, physical examination, and vaginal (or cervical if cervix remains) cytology, but not imaging. Surveillance frequency for symptoms review and examination is dependent upon stage and adjuvant therapy. For lower-risk women (early stage, treated surgically), a visit every 6 months for the first 2 years and then yearly is acceptable. For high-risk women (advanced stage, chemotherapy, radiation, or both, plus surgery), visits every 3 months for the first 2 years, followed by every 6 months for another 3 years, then annually is recommended. For both groups, yearly vaginal (or cervical if cervix remains) cytology is advised. CT, PET, or both are recommended only for suspected recurrence. ${ }^{11}$

Given the lack of prospective data, Cancer Care Ontario (CCO) published practice guidelines for posttreatment cervical cancer surveillance based on retrospective studies and expert consensus. A Cochrane review similarly found no evidence to guide decision-making. ${ }^{16} \mathrm{CCO}$ concluded that visits to update history and perform a pelvic examination are warranted every 3-4 months for 2 years, every 6-12 months during years $3-5$, then annually. In contrast to the SGO, the 
CCO group considered the value of vaginal cytology for recurrence detection minimal. Cytology would detect new (not recurrent) precancer and should be performed at most annually. ${ }^{17}$

\section{Vulvar and vaginal cancer surveillance}

Evidence supporting recommendations for surveillance following vaginal or vulvar cancer is even more limited given the low incidence of these malignancies. Guidelines from the SGO for vulvar and vaginal cancer follow up are the same as for cervical cancer (see Fig. 1). ${ }^{11}$

The American College of Obstetricians and Gynecologists emphasizes that these women are at high risk of vulvar intraepithelial lesions and recurrent vulvar cancer. Although evidence-based guidelines are lacking, they recommend pelvic examination at 6 and 12 months after treatment, and annually thereafter. ${ }^{18}$

A study of vulvar cancer reported a higher recurrence rate within 2 years after treatment in women with inguinal lymph node involvement $(32.7 \%)$ versus those without $(5.1 \%)$. However, later recurrence rates were similar in both groups (2.7\% and $2.8 \% 2-5$ years after initial treatment; $8.8 \%$ and $9.7 \%$ at $>5$ years). The importance of long-term surveillance following vulvar cancer treatment was illustrated by the $9.7 \%$ of women with late recurrence. ${ }^{19}$

\section{Management of Early Menopause Related to Cancer Treatment}

\section{Hormonal treatment}

Menopause may occur spontaneously in the gynecologic cancer survivor, or may be consequent to cancer treatment (chemotherapy, radiation, or bilateral salpingo-oophorectomy [BSO]). An abrupt transition can result in severe vasomotor symptoms (VMS), sleep disturbance, cognitive issues, joint pain, genitourinary syndrome of menopause (GSM), and sexual dysfunction. The safety of hormone therapy (HT) using estrogen or estrogen plus a progestogen in the setting of a gynecologic cancer is a concern for patients and providers alike. However, global fears about HT-related recurrence risk and decreased survival are not uniformly evidence supported. Hormone therapy is the most effective treatment for VMS, which occur in about $75 \%$ of menopausal women. ${ }^{20}$ Although HT should be avoided in the setting of some gynecologic cancers, it can be used safely in many women. Maintaining quality of life for cancer survivors includes minimizing the physical and psychological impact of cancer. Management of VMS and GSM with HT can mitigate the impact of cancer and its treatment on quality of life.

\section{Endometrial cancer}

Increased estrogen exposure is a risk factor for endometrioid-type endometrial carcinoma, which accounts for about $90 \%$ of endometrial cancer cases. ${ }^{21}$ Regimens of HT using unopposed estrogen or estrogen with a progestogen administered cyclically have been associated with an increased risk of endometrial cancer. ${ }^{22,23}$ However, more recent studies of combination HT using estrogen plus a progestogen, either in a cyclic or continuous manner, have either failed to show an increased risk ${ }^{24}$ or have been associated with a decreased risk of endometrial cancer. ${ }^{25}$ Because endometrial cancer is usually identified at an early stage and is associated with an $85 \%$ survival rate, overall quality of life for long-term survivors is a crucial consideration. In fact, about $20 \%$ of women are premenopausal at diagnosis, with $5 \%$ under the age of 40 years. $^{26}$

Though intuitively estrogen should be contraindicated for survivors of endometrial carcinoma, existing data does not suggest an increased risk of recurrence or mortality with HT. ${ }^{27-30}$ Furthermore, some studies note a decreased recurrence risk or even a survival advantage with $\mathrm{HT} .{ }^{28-30}$ While two prospective studies showed no evidence of increased recurrence risk with HT, significant limitations flawed each study. ${ }^{31,32}$ A recent meta-analysis did not show an association between HT use and risk of endometrial cancer recurrence in early stage disease, though the authors suggest additional randomized, controlled trials are needed to confirm these results. ${ }^{33}$

In contrast to data suggesting that HT in survivors of early stage endometrial cancer is not detrimental, little evidence supports the safety of HT in the setting of advanced disease, wherein concerns exist regarding potential estrogen stimulation of residual tumor cells. Similarly, data regarding HT in women with a history of uterine sarcomas (leiomyosarcoma, endometrial stromal sarcoma, undifferentiated endometrial sarcoma, and carcinosarcoma) are lacking. ${ }^{26,34}$

\section{Ovarian cancer}

The majority of women with ovarian cancer undergo BSO with consequent menopause if they were previously premenopausal. Women who used HT after epithelial ovarian cancer diagnosis had no change in disease-free survival in a retrospective $^{35}$ and a subsequent randomized study, ${ }^{36}$ and no survival disadvantage or a potential survival advantage in three additional studies. ${ }^{37-39}$ While sex cord and germ cell tumors represent only $8 \%$ of ovarian cancers, they often present in young women at an early stage. They are generally treated with unilateral oophorectomy and chemotherapy. ${ }^{26}$ If chemotherapy renders the remaining ovary nonfunctional, HT is thought safe in survivors of germ cell tumors, although definitive data are lacking. In the setting of granulosa cell tumors, HT is not recommended given concerns about hormone-dependent tumor stimulation and risk of recurrence. $^{21,26,40}$

\section{Cervical, vulvar, and vaginal cancers}

Cervical, vulvar, and vaginal cancers are almost exclusively related to high-risk HPV and are not hormone dependent. Therefore, HT is not contraindicated. About $80 \%$ of cervical cancers are squamous cell cancers, which are not hormone dependent. ${ }^{26}$ In contrast, about $15 \%$ of cervical cancers are adenocarcinomas, which may be linked to estrogen exposure. However, no evidence links HT in cervical adenocarcinoma survivors with increased risk of recurrence or reduced disease-free survival. ${ }^{21,26,34,40}$ Vaginal cancers are most commonly squamous cell cancers associated with HPV and not hormone dependent. ${ }^{21}$ Vaginal clear cell adenocarcinoma is extremely rare, with most cases diagnosed in women with diethylstilbestrol (DES) exposure in utero. There are no data on HT in this population, though many have passed the age of menopause. ${ }^{21,34}$ 


\section{Nonhormonal treatment of vasomotor symptoms}

After BSO, both premenopausal and postmenopausal women experience an increase in VMS, which can negatively impact quality of life. The occurrence of VMS is attributed to decreased endogenous estrogen and androgens. ${ }^{41}$ It is paramount that physicians caring for gynecologic cancer survivors educate women on the potential risks and benefits of hormonal and nonhormonal treatments. ${ }^{42}$

While nonhormonal therapies are often recommended, they are not as effective as HT in reducing VMS. Strategies such as paced respirations and avoidance of triggers for VMS such as caffeine, alcohol, and tobacco may be helpful. ${ }^{43,44}$ Studies regarding the effect of exercise on VMS report inconsistent results, although exercise is associated with improved quality of life, reduced stress, improvement in mood and somatic complaints, and less weight gain and muscle loss. It is also cost effective and has few side effects. ${ }^{45}$ Complementary and integrative therapies including black cohosh and phytoestrogens have not shown convincing evidence of efficacy for VMS. ${ }^{46,47}$ While a Cochrane review including 16 randomized, controlled trials concluded that there is insufficient evidence that acupuncture is effective for VMS, a recent meta-analysis of 12 trials suggested that acupuncture is associated with reduced VMS frequency and severity, as well as improvement in menopause-related symptoms and quality of life. Interestingly, sham acupuncture was shown to be equally effective. ${ }^{48,49}$ Selective norepinephrine reuptake inhibitors and selective serotonin reuptake inhibitors such as venlafaxine and paroxetine, respectively, are associated with modest VMS improvement but also have the potential for adverse sexual side effects. ${ }^{50}$ Low-dose paroxetine $7.5 \mathrm{mg}$ was found to reduce VMS frequency and severity and is the first non-hormonal treatment for VMS approved by the FDA. ${ }^{51}$ Gabapentin is modestly effective for VMS in doses up to $900 \mathrm{mg}$ daily. ${ }^{52}$

\section{Bone health}

Women experiencing premature menopause and estrogen deprivation following $\mathrm{BSO}$ are at higher risk for osteopenia and osteoporosis. A study of BRCA mutation carriers who underwent BSO before age 50 years demonstrated increased rates of osteopenia (62\%) and osteoporosis (9\%). ${ }^{53}$ Although national guidelines are lacking for postsurgical care of gynecologic cancer survivors, there are guidelines for management of bone health among breast cancer survivors. ${ }^{54,55}$

The United Kingdom Expert Group developed guidelines for women with treatment-induced menopause. For women under age 45 years, a bone mineral density measurement of the spine and hip by dual energy x-ray absorptiometry (DXA) is recommended at baseline. If the T-score is -1.0 or higher, no additional testing is recommended. If the T-score is less than -2.0 , bisphosphonate treatment is suggested, followed by a repeat DXA in 24 months. If the T-score is between -1.0 and -2.5 , a repeat DXA is advised in 24 months. ${ }^{55}$

All guidelines emphasize counseling regarding adequate dietary or supplemental intake of calcium (1200 mg elemental calcium per day) and vitamin D (800-1000 IU per day). Further, women are encouraged to participate in regular weight-bearing exercise, stop smoking, and avoid excessive alcohol. ${ }^{54,55}$

\section{Cardiovascular health}

While BSO prior to the natural age of menopause may be necessary for treatment of gynecologic cancer, it is unfortunately associated with increased mortality risk, primarily from coronary heart disease (CHD), ${ }^{56,57}$ even when performed in women up to 55 years of age. ${ }^{58}$ Total abdominal hysterectomy is also associated with increased CHD mortality, but the risk is lower than that following $\mathrm{BSO}$, and attenuates with advancing age. ${ }^{58}$ Increased CHD mortality risk is thought to be mitigated by estrogen treatment. ${ }^{56,57}$ Counseling women regarding individual cardiovascular risk and modification of other CHD risk factors is important.

\section{Brain health}

Women with early menopause following BSO may not only have increased risk of severe VMS, osteoporosis, CHD, and sexual dysfunction, they may also have an increased risk of cognitive impairment or dementia and parkinsonism, and may benefit from HT at least until the average age of natural menopause (around age 51 years). ${ }^{59-61}$

\section{Fertility preservation}

As chemotherapeutic agents for gynecologic malignancy treatment may result in temporary or permanent subfertility, the American Society of Clinical Oncology (ASCO) published recommendations suggesting that all newly-diagnosed patients desiring future fertility be offered a consultation regarding fertility preservation. ${ }^{62}$ Therefore, a discussion with a reproductive endocrinologist should be included in the management of women diagnosed with gynecologic cancer not involving both ovaries. Fertility preservation counseling should emphasize the potential effect of cancer treatment on future fertility, as well as options for fertility preservation and parenthood after cancer. In some cases, patients should be encouraged to obtain legal assistance for disposition of stored gametes, embryos, and tissue in the event of death. ${ }^{63}$

In a recent review, gynecologic cancer survivors, though older, had a greater rate of success with subsequent in vitro fertilization than other groups, including breast, hematologic, and other cancer survivors. ${ }^{64}$ Cryopreservation and autotransplantation of ovarian tissue has been associated with restoration of fertility and endocrine function and did not delay chemotherapy, even in the setting of hormone-dependent cancer. ${ }^{65}$

\section{Contraception}

Many women treated for gynecologic cancer during their reproductive years undergo hysterectomy with or without BSO as part of treatment, therefore obviating the need for future contraception. However, the incidence of chemotherapy-induced amenorrhea in patients without hysterectomy and $\mathrm{BSO}$, reported to be $53 \%-89 \%$, is more likely reversible in women under 40 years than in older women. ${ }^{66}$

In this setting, a diagnosis of cancer or desire for future childbearing should not exclude a contraception consultation, ideally with a provider with experience and expertise in contraception in the setting of gynecologic cancer. Optimally, the contraceptive method recommended would be consistent with U.S. Medical Eligibility Criteria ${ }^{67}$ and might serve as part of treatment. For example, the levonorgestrel- 
releasing intrauterine system (LNG IUS) has been successfully used in the setting of grade 1 , stage 1 endometrial cancer and endometrial hyperplasia with atypia in young women to not only treat the endometrial lesion, but also to provide effective and convenient contraception. ${ }^{68-70}$

Patients with estrogen-dependent cancer are encouraged to choose estrogen-free contraception such as long-acting reversible contraception, which includes the LNG IUS, copper intrauterine device, and contraceptive implant. Women with non-estrogen-dependent cancers can choose any convenient and acceptable method. The key is to avoid the assumption that patients are not sexually active or are permanently infertile as a result of cancer treatment.

\section{Sexual health}

Sexual health may be negatively impacted by gynecologic cancer and its treatment. Impairment in sexual functioning may result from hormone loss as well as alterations in pelvic anatomy. In premenopausal women, hormone loss with BSO involves a loss of both estrogens and androgens. Likewise, postmenopausal women also lose androgens, even many years after menopause. ${ }^{71}$ Because of rapid cell turnover, pelvic radiation may have an acute effect on vulvar and vaginal tissues, including desquamation. Radiation effects may progress over time, resulting in vaginal dryness, thinning, adhesions, fibrosis, reduced elasticity, and consequently, vaginal shortening and narrowing. ${ }^{72}$ Chemotherapy may result in mucositis involving the vaginal tissues. ${ }^{73}$ The diagnosis of cancer and its treatment can also lead to changes in body image that may adversely affect sexual function.

Despite the high prevalence of sexual dysfunction in gynecologic cancer survivors, a survey of gynecologic oncologists revealed that fewer than $50 \%$ routinely asked patients about sexual health, and $80 \%$ felt they had inadequate time to address sexual health concerns. ${ }^{74}$ The ideal time to address such concerns may vary depending on tumor site, prognosis, and treatment modalities. ${ }^{75}$

Sexual dysfunction in gynecologic cancer survivors is often multifactorial and best managed using a multidisciplinary approach. A psychologist or sex therapist can manage the psychological, interpersonal, and sociocultural factors contributing to sexual dysfunction, including fear of cancer recurrence, anxiety, depression, concerns about body image, sexual pain, and relationship factors. A physical therapist with expertise in pelvic floor muscle dysfunction can improve kinesthetic awareness, facilitate relaxation of pelvic floor muscles, reduce pain associated with vaginal penetration or examination, and ultimately strengthen weakened muscles in those with musculoskeletal pain. The primary healthcare provider, oncologist, or gynecologist may focus on the medical aspects of sexual dysfunction in the survivor of gynecologic cancer, such as GSM, provoked vestibulodynia, or vaginal shortening or stenosis. Vaginal lubricants, moisturizers, local vaginal estrogen therapy (cream, ring, or tablet), and the selective estrogen receptor modulator, ospemifene, can be used for the management of GSM. Vaginal dilators and the use of a vibrator may also be helpful for maintaining vaginal health. ${ }^{76}$

\section{Genetic counseling and testing}

Genetic testing to identify a genetic predisposition to malignancy is indicated when cancer is diagnosed in a young woman or in the setting of multiple affected relatives. A detailed family history including identification of relatives with a history of breast, ovarian, endometrial, or other potentially hereditary malignancy should be obtained. A genetic counselor can review risks and benefits of available testing options for appropriate candidates. ${ }^{77,78}$

\section{Conclusions}

Because the 5-year survival rate for most gynecologic cancers is greater than $65 \%$, the care of women expands beyond their cancer diagnosis to include issues concerning the transition from cancer treatment to surveillance, survivorship, quality of life, and long-term preventive care. Although evidence for specific surveillance recommendations is sometimes limited to retrospective studies and expert opinion, clinicians can rely on SGO, ASCO, and other consensus group recommendations for the various gynecologic cancers. With extended survivorship, concerns regarding fertility, cardiovascular, bone, brain, and sexual health, as well as management of the effects of cancer treatment become an important component of care. Much of this care can be provided in the primary care setting in collaboration with a woman's oncologist.

\section{Acknowledgment}

Kristi Simmons, Research and Academic Support Services, Mayo Clinic, formatted and proofread the manuscript.

\section{Author Disclosure Statement}

Drs. Long and Casey are certified contraceptive implant (Nexplanon) trainers and receive research grant support from Merck. Drs. Pruthi, MacLaughlin, and Faubion report no competing financial interests.

\section{References}

1. American Cancer Society. Cancer Facts and Figures 2015. Atlanta: American Cancer Society; 2015.

2. Surveillance, Epidemiology, and End Results (SEER) Program of the National Cancer Institute (NCI). SEER cancer statistics factsheets: Cervix uteri cancer. Available at: http:// seer.cancer.gov/statfacts/html/cervix.html Accessed September 29, 2014.

3. SEER, NCI. SEER cancer statistics factsheets: Endometrial cancer. Available at: http://seer.cancer.gov/statfacts/html/ corp.html Accessed September 29, 2014.

4. SEER, NCI. SEER cancer statistics factsheets: Ovary cancer. Available at: http://seer.cancer.gov/statfacts/html/ ovary.html Accessed September 29, 2014.

5. SEER, NCI. SEER cancer statistics factsheets: Vulvar cancer. Available at: http://seer.cancer.gov/statfacts/html/ vulva.html Accessed September 29, 2014.

6. Combination oral contraceptive use and the risk of endometrial cancer. The Cancer and Steroid Hormone Study of the Centers for Disease Control and the National Institute of Child Health and Human Development. JAMA 1987;257: 796-800.

7. Isin Dogan Ekici A, Kucukali T, Coskun Salman M, Ayhan A. Triple simultaneous primary gynecological malignancies in a 56-year-old patient. Int J Gynecol Cancer 2006; 16:1947-1950. 
8. Vang R, Shih Ie M, Kurman RJ. Fallopian tube precursors of ovarian low- and high-grade serous neoplasms. Histopathology 2013;62:44-58.

9. Leyden WA, Manos MM, Geiger AM, et al. Cervical cancer in women with comprehensive health care access: Attributable factors in the screening process. J Natl Cancer Inst 2005;97:675-683.

10. Cancer.Net. About Cancer Survivorship. Available at: www.cancer.net/survivorship/about-cancer-survivorship. Accessed February 10, 2015.

11. Salani R, Backes FJ, Fung MF, et al. Posttreatment surveillance and diagnosis of recurrence in women with gynecologic malignancies: Society of Gynecologic Oncologists recommendations. Am J Obstet Gynecol 2011;204:466-478.

12. Lalwani N, Dubinsky T, Javitt MC, et al. ACR Appropriateness Criteria $(\mathrm{R})$ pretreatment evaluation and followup of endometrial cancer. Ultrasound Q 2014;30:21-28.

13. Sartori E, Pasinetti B, Chiudinelli F, et al. Surveillance procedures for patients treated for endometrial cancer: A review of the literature. Int J Gynecol Cancer 2010;20: 985-992.

14. Kew F, Galaal K, Bryant A, Naik R. Evaluation of followup strategies for patients with epithelial ovarian cancer following completion of primary treatment. Cochrane Database Syst Rev 2011:CD006119.

15. Piovano E, Attamante L, Macchi C, et al. The role of HE4 in ovarian cancer follow-up: A review. Int J Gynecol Cancer 2014;24:1359-1365.

16. Lanceley A, Fiander A, McCormack M, Bryant A. Followup protocols for women with cervical cancer after primary treatment. Cochrane Database Syst Rev 2013;11:CD008767.

17. Elit L, Fyles AW, Oliver TK, Devries-Aboud MC, FungKee-Fung M. Follow-up for women after treatment for cervical cancer. Curr Oncol 2010;17:65-69.

18. American College of Obstetricians and Gynecologists. ACOG committee opinion No. 509: Management of vulvar intraepithelial neoplasia. Obstet Gynecol 2011;118:1192-1194.

19. Gonzalez Bosquet J, Magrina JF, Gaffey TA, et al. Longterm survival and disease recurrence in patients with primary squamous cell carcinoma of the vulva. Gynecol Oncol 2005;97:828-833.

20. Utian WH. Psychosocial and socioeconomic burden of vasomotor symptoms in menopause: A comprehensive review. Health Qual Life Outcomes 2005;3:47.

21. Singh P, Oehler MK. Hormone replacement after gynaecological cancer. Maturitas 2010;65:190-197.

22. Beresford SA, Weiss NS, Voigt LF, McKnight B. Risk of endometrial cancer in relation to use of oestrogen combined with cyclic progestagen therapy in postmenopausal women. Lancet 1997;349:458-461.

23. Weiderpass E, Adami HO, Baron JA, et al. Risk of endometrial cancer following estrogen replacement with and without progestins. J Natl Cancer Inst 1999;91:1131-1137.

24. Lacey JV, Jr., Leitzmann MF, Chang SC, et al. Endometrial cancer and menopausal hormone therapy in the National Institutes of Health-AARP Diet and Health Study cohort. Cancer 2007;109:1303-1311.

25. Archer DF. The effect of the duration of progestin use on the occurrence of endometrial cancer in postmenopausal women. Menopause 2001;8:245-251.

26. Biliatis I, Thomakos N, Rodolakis A, Akrivos N, Zacharakis D, Antsaklis A. Safety of hormone replacement therapy in gynaecological cancer survivors. J Obstet Gynaecol 2012;32:321-325.
27. Chapman JA, DiSaia PJ, Osann K, Roth PD, Gillotte DL, Berman ML. Estrogen replacement in surgical stage I and II endometrial cancer survivors. Am J Obstet Gynecol 1996;175: 1195-1200.

28. Creasman WT, Henderson D, Hinshaw W, Clarke-Pearson DL. Estrogen replacement therapy in the patient treated for endometrial cancer. Obstet Gynecol 1986;67:326-330.

29. Lee RB, Burke TW, Park RC. Estrogen replacement therapy following treatment for stage I endometrial carcinoma. Gynecol Oncol 1990;36:189-191.

30. Suriano KA, McHale M, McLaren CE, Li KT, Re A, DiSaia PJ. Estrogen replacement therapy in endometrial cancer patients: A matched control study. Obstet Gynecol 2001;97:555-560.

31. Ayhan A, Taskiran C, Simsek S, Sever A. Does immediate hormone replacement therapy affect the oncologic outcome in endometrial cancer survivors? Int J Gynecol Cancer 2006;16: 805-808.

32. Barakat RR, Bundy BN, Spirtos NM, Bell J, Mannel RS. Randomized double-blind trial of estrogen replacement therapy versus placebo in stage I or II endometrial cancer: A Gynecologic Oncology Group Study. J Clin Oncol 2006;24:587-592.

33. Shim SH, Lee SJ, Kim SN. Effects of hormone replacement therapy on the rate of recurrence in endometrial cancer survivors: A meta-analysis. Eur J Cancer 2014;50:16281637.

34. Guidozzi F. Estrogen therapy in gynecological cancer survivors. Climacteric 2013;16:611-617.

35. Eeles RA, Tan S, Wiltshaw E, et al. Hormone replacement therapy and survival after surgery for ovarian cancer. BMJ 1991;302:259-262.

36. Guidozzi F, Daponte A. Estrogen replacement therapy for ovarian carcinoma survivors: A randomized controlled trial. Cancer 1999;86:1013-1018.

37. Bebar S, Ursic-Vrscaj M. Hormone replacement therapy after epithelial ovarian cancer treatment. Eur J Gynaecol Oncol 2000;21:192-196.

38. Mascarenhas C, Lambe M, Bellocco R, et al. Use of hormone replacement therapy before and after ovarian cancer diagnosis and ovarian cancer survival. Int $\mathbf{J}$ Cancer 2006;119:2907-2915.

39. Ursic-Vrscaj M, Bebar S, Zakelj MP. Hormone replacement therapy after invasive ovarian serous cystadenocarcinoma treatment: The effect on survival. Menopause 2001;8:70-75.

40. Hinds L, Price J. Menopause, hormone replacement and gynaecological cancers. Menopause Int 2010;16:89-93.

41. Sluijmer AV, Heineman MJ, De Jong FH, Evers JL. Endocrine activity of the postmenopausal ovary: The effects of pituitary down-regulation and oophorectomy. J Clin Endocrinol Metab 1995;80:2163-2167.

42. Madalinska JB, van Beurden M, Bleiker EM, et al. The impact of hormone replacement therapy on menopausal symptoms in younger high-risk women after prophylactic salpingo-oophorectomy. J Clin Oncol 2006;24:3576-3582.

43. Faubion SS, Sood R, Thielen JM, Shuster LT. Caffeine and menopausal symptoms: What is the association? Menopause 2015;22:155-158.

44. Sood R, Sood A, Wolf SL, et al. Paced breathing compared with usual breathing for hot flashes. Menopause 2013;20: 179-184.

45. Stojanovska L, Apostolopoulos V, Polman R, Borkoles E. To exercise, or, not to exercise, during menopause and beyond. Maturitas 2014;77:318-323. 
46. Leach MJ, Moore V. Black cohosh (Cimicifuga spp.) for menopausal symptoms. Cochrane Database Syst Rev 2012;9: CD007244.

47. Lethaby A, Marjoribanks J, Kronenberg F, Roberts H, Eden J, Brown J. Phytoestrogens for menopausal vasomotor symptoms. Cochrane Database Syst Rev 2013;12:CD001395.

48. Chiu HY, Pan CH, Shyu YK, Han BC, Tsai PS. Effects of acupuncture on menopause-related symptoms and quality of life in women on natural menopause: A meta-analysis of randomized controlled trials. Menopause 2014;22:234-244.

49. Dodin S, Blanchet C, Marc I, et al. Acupuncture for menopausal hot flushes. Cochrane Database Syst Rev 2013;7:CD007410.

50. Shams T, Firwana B, Habib F, et al. SSRIs for hot flashes: A systematic review and meta-analysis of randomized trials. J Gen Intern Med 2014;29:204-213.

51. Simon JA, Portman DJ, Kaunitz AM, et al. Low-dose paroxetine $7.5 \mathrm{mg}$ for menopausal vasomotor symptoms: Two randomized controlled trials. Menopause 2013;20:1027-1035.

52. Butt DA, Lock M, Lewis JE, Ross S, Moineddin R. Gabapentin for the treatment of menopausal hot flashes: A randomized controlled trial. Menopause 2008;15:310-318.

53. Cohen JV, Chiel L, Boghossian L, et al. Non-cancer endpoints in BRCA1/2 carriers after risk-reducing salpingooophorectomy. Fam Cancer 2012;11:69-75.

54. Hillner BE, Ingle JN, Chlebowski RT, et al. American Society of Clinical Oncology 2003 update on the role of bisphosphonates and bone health issues in women with breast cancer. J Clin Oncol 2003;21:4042-4057.

55. Reid DM, Doughty J, Eastell R, et al. Guidance for the management of breast cancer treatment-induced bone loss: A consensus position statement from a UK Expert Group. Cancer Treat Rev 2008;34:S3-S18.

56. Parker WH, Broder MS, Chang E, et al. Ovarian conservation at the time of hysterectomy and long-term health outcomes in the nurses' health study. Obstet Gynecol 2009;113:1027-1037.

57. Rivera CM, Grossardt BR, Rhodes DJ, et al. Increased cardiovascular mortality after early bilateral oophorectomy. Menopause 2009;16:15-23.

58. Gierach GL, Pfeiffer RM, Patel DA, et al. Long-term overall and disease-specific mortality associated with benign gynecologic surgery performed at different ages. Menopause 2014;21:592-601.

59. Rocca WA, Bower JH, Maraganore DM, et al. Increased risk of cognitive impairment or dementia in women who underwent oophorectomy before menopause. Neurology 2007;69:1074-1083.

60. Rocca WA, Bower JH, Maraganore DM, et al. Increased risk of parkinsonism in women who underwent oophorectomy before menopause. Neurology 2008;70:200-209.

61. Rocca WA, Grossardt BR, Shuster LT. Oophorectomy, estrogen, and dementia: A 2014 update. Mol Cell Endocrinol 2014;389:7-12.

62. Lee SJ, Schover LR, Partridge AH, et al. American Society of Clinical Oncology recommendations on fertility preservation in cancer patients. J Clin Oncol 2006;24:2917-2931.

63. Jensen JR, Morbeck DE, Coddington CC, 3rd. Fertility preservation. Mayo Clin Proc 2011;86:45-49.

64. Pavone ME, Hirshfeld-Cytron J, Lawson AK, Smith K, Kazer R, Klock S. Fertility preservation outcomes may differ by cancer diagnosis. J Hum Reprod Sci 2014;7:111-118.
65. Gamzatova Z, Komlichenko E, Kostareva A, et al. Autotransplantation of cryopreserved ovarian tissue - effective method of fertility preservation in cancer patients. Gynecol Endocrinol 2014;30 Suppl 1:43-47.

66. Di Cosimo S, Alimonti A, Ferretti G, et al. Incidence of chemotherapy-induced amenorrhea depending on the timing of treatment by menstrual cycle phase in women with early breast cancer. Ann Oncol 2004;15:1065-1071.

67. Centers for Disease Control and Prevention (CDC). The United States medical eligibility criteria for contraceptive use, 2010. MMWR 2010;59(RR-4):1-85. Available at: www.cdc.gov/ reproductivehealth/unintendedpregnancy/usmec.htm. Accessed September 28, 2014.

68. Gallos ID, Krishan P, Shehmar M, Ganesan R, Gupta JK. LNG-IUS versus oral progestogen treatment for endometrial hyperplasia: A long-term comparative cohort study. Hum Reprod 2013;28:2966-2971.

69. Kudesia R, Singer T, Caputo TA, et al. Reproductive and oncologic outcomes after progestin therapy for endometrial complex atypical hyperplasia or carcinoma. Am J Obstet Gynecol 2014;210:255.e1-4.

70. Gunderson CC, Fader AN, Carson KA, Bristow RE. Oncologic and reproductive outcomes with progestin therapy in women with endometrial hyperplasia and grade 1 adenocarcinoma: A systematic review. Gynecol Oncol 2012;125: 477-482.

71. Davison SL, Bell R, Donath S, Montalto JG, Davis SR. Androgen levels in adult females: Changes with age, menopause, and oophorectomy. J Clin Endocrinol Metab 2005;90:3847-3853.

72. Incrocci L, Jensen PT. Pelvic radiotherapy and sexual function in men and women. J Sex Med 2013;10(Suppl 1): 53-64.

73. Krychman ML, Carter J, Aghajanian CA, Dizon DS, Castiel M. Chemotherapy-induced dyspareunia: A case study of vaginal mucositis and pegylated liposomal doxorubicin injection in advanced stage ovarian carcinoma. Gynecol Oncol 2004;93:561-563.

74. Wiggins DL, Wood R, Granai CO, Dizon DS. Sex, intimacy, and the gynecologic oncologists: Survey results of the New England Association of Gynecologic Oncologists (NEAGO). J Psychosoc Oncol 2007;25:61-70.

75. Falk SJ, Dizon DS. Sexual dysfunction in women with cancer. Fertil Steril 2013;100:916-921.

76. Management of symptomatic vulvovaginal atrophy: 2013 position statement of The North American Menopause Society. Menopause 2013;20:888-902, quiz 903-884.

77. ACOG Practice Bulletin No. 103: Hereditary breast and ovarian cancer syndrome. Obstet Gynecol 2009;113:957-966.

78. Christinat A, Pagani O. Practical aspects of genetic counseling in breast cancer: Lights and shadows. Breast 2013;22: $375-382$

Address correspondence to: Stephanie S. Faubion, MD Division of General Internal Medicine Mayo Clinic 200 First Street Southwest Rochester, MN 55905

E-mail: Faubion.Stephanie@mayo.edu 